\title{
Suicide Prevention in Mental Health Services- A Qualitative Study of a Web Based Program for Mental Health Care Staff
}

\author{
Sigrid Stjernswärd, Lars Hansson \\ Department of Health Sciences, Lund University, Lund, Sweden \\ Email: sigrid.stjernsward@med.lu.se
}

Received August 21, 2013; revised September 21, 2013; accepted September 28, 2013

Copyright (c) 2013 Sigrid Stjernswärd, Lars Hansson. This is an open access article distributed under the Creative Commons Attribution License, which permits unrestricted use, distribution, and reproduction in any medium, provided the original work is properly cited.

\begin{abstract}
Background: Further strategies are needed to deal with the high losses to suicide. New modalities should be explored within the context of suicide prevention. Aim: The aim of the study was to evaluate participants' experiences of a web based program for mental health care staff, including its potential clinical relevance. Methods: Nineteen participants participated in five focus groups. Data was analyzed using content analysis. Results: The analysis showed participants' experiences of the program's contents and format ("Web Based Modules", "Discussion Groups") and practical value ("Clinical Relevance and Use", "Effects on Communication and Climate”). Conclusions: The program partly increased awareness about risk factors and the importance of inquiring about suicide ideation/plans and documenting suicide assessments. Experiences of the clinical value were varying and may be increased through potential enhancements.
\end{abstract}

Keywords: Competence; Psychiatric Services; Mental Health Care Staff; Suicide Prevention; Web Based Program

\section{Introduction}

Losses to suicide are estimated at 877,000 persons per year [1]. Suicide is a prominent cause of death in youth standing for $6 \%$ of the 2.6 million deaths among young people in 2004 [2]. Suicidal behavior is mainly caused by proximal stressors or triggers and predisposition [3]. Psychiatric illnesses are a major contributor in over $90 \%$ of suicides and over $80 \%$ of cases are untreated at time of death [4]. Up to $83 \%$ of patients have been in contact with a primary care physician within a year of their death by suicide and up to $66 \%$ within a month $[5,6]$. Depression can be treated in primary care, but lack of resources and stigma are known barriers to treatment [7]. Further factors contribute to suicide, including the availability of lethal means, alcohol and drug abuse, attitudes to suicide, help-seeking behavior, physical illness, marital status, age and sex [3]. Approximately 1200 persons/year commit suicide in Sweden. About half of them had contact with health care within a month prior to their death [8].

Systematic reviews show effective strategies to prevent suicide, although further studies are needed to evaluate key components, cost-effectiveness and potential synergistic effects of multilevel interventions [3,9], measuring both primary (reduced suicides) and seconddary (e.g., increased help-seeking and treatment of depression) outcomes. Education of primary care physicians to recognize and treat depression and the restriction of access to lethal means were identified as effective interventions $[3,9]$. Improving accessibility of care is central [9]. Guidelines rule care professionals' work in clinical practice and the support and care given to patients [10,11]. Regional and local contexts are also influenced by local regulations and organizational structures, including individual leadership styles [12]. The context can be decisive for implementation outcomes, including barriers such as resistance on managers' level, innovation complexity and resources [13]. Insufficient managerial support is a known obstacle to implementation of suicide prevention activities [14]. Psychiatric contexts are characterized by specific organizational structures and routines that are not always compatible with a "learning organization", e.g. an attitude of risk minimization and skepticism towards innovations and new solutions to old problems, which can inhibit implementation success [13].

The literature shows that education is one of the most 
frequently used implementation strategies within health care research [15]. However the evidence for its effectiveness is still weak, partly due to the challenge of evaluating and comparing studies and the lack of a theoretical base. A comparative study of a suicide intervention training program for mental health services staff showed that a more complex training intervention focusing on creating and strengthening networks led to better knowledge and significantly higher number of organizational links than a standard training model [16]. Despite its limitations, the study showed that a multifaceted approach can be more effective for a successful implementation of innovations [16].

\section{Suicide Prevention in Scanian Mental Health Services (SPiSS)}

The number of (possible) suicides in connection with care within mental health services decreased in Scaniaa province in southern Sweden-from 51 in 2010 to 37 in 2012 [17]. Scanian Mental Health Services (SMS) is the regional administration for mental health services in the region, acting as second line services. The reason for the drop in suicide is not known, but one hypothesis is the mental health services' focus on suicide prevention, including the initiative Suicide Prevention in Scanian Mental Health Services (SPiSS). SPiSS is a web based program with the aim of decreasing the number of suicide attempts. It consists of fact modules, knowledge tests, dialogues at work and certification of units of care. The fact modules are performed individually with a computer. Simulated patient cases are discussed in group meetings, during which care units/teams discuss how they would act in similar situations.

The program's underpinnings focus on patient safety, good treatment, security and knowledge. The vision is an enhanced safety for patients and families together with committed and competent staff. Keywords are: dare to ask, document, treat well, communicate and act. The program is aimed at all staff within SMS. The target group consists of 2907 staff with diverse professions. The main groups consist of physicians (310), social workers (268), psychologists (278), nurses (744) and assistant nurses (787). Additional groups are occupational therapists, physiotherapists, secretarial staff and administrative staff. The concerned units of care within SMS will be certified between November 2012 and December 2013. Certification of the units is obtained through completion of the web based program and participation in group meetings. SMS's vision is for $80 \%$ of the units' staff to be certified.

The web based program entails four modules, out of which the first two are compulsory, which all staff is expected to complete. The respective modules contain facts about suicide statistics, risk and protective factors, explicative models, and assessment instruments. There are totally four group meetings/year led by a discussion leader (2 meetings/semester). They are compulsory only for staff working close to patients. Approximately sixteen group leaders are initially responsible for leading the group discussions. The plan is for SPiSS to become a compulsory program for all staff within SMS.

The aim of the present study was to evaluate participants' experiences of SPiSS and of its potential clinical relevance and practical use.

\section{Methods}

\subsection{Design and Data Collection}

A qualitative design was chosen for the present evaluation study. Data was collected through five focus groups including three to five persons per group (total $n=19$ ) using a semi-structured interview guide [18]. The focus groups were led by a moderator [19]. To get an understanding of SPiSS the moderator carried out the web based modules and participated as an observer in a meeting between group leaders and in a group discussion amongst staff participating in a SPiSS discussion group.

Data collection was carried out at clinics in four main municipalities within Scania. Prior to the focus group, participants answered a short questionnaire to collect information about gender, age, profession and current position, work experience within mental health care, and number of completed modules and attended discussion groups. The interviews lasted approximately 55 minutes each. They were recorded digitally and transcribed verbatim for analysis.

\subsection{Sample}

Information about the study was spread through managerial levels in SMS to heads of units from a number of psychiatric units within the concerned municipalities. The information was forwarded to their respective staff. Those fulfilling the inclusion criteria that were able to attend a focus group on one of a number of predetermined dates participated in the study. Inclusion criteria were being employed by SMS, having carried out at least two out of four web based modules, and at least one group discussion at the time of the interview. Inclusion in the sample was planned according to the principle of maximal variation to get a representative sample in terms of age, gender, profession, professional experience from working within psychiatric care, and type of care unit. The focus groups' composition was hence done according to a heterogeneity principle to illuminate the views of participants with different backgrounds. This also permitted comparisons within and across groups [20,21]. 
After the fifth group no new information appeared, speaking for saturation of information.

In total nineteen persons participated, out of which 12 were women and 7 men. Participants were aged 25 - 65 (mean age $=47$ ). The represented professions were: nurse (8, out of which one had a psychiatry specialization), social worker (3), physician (1), psychologist (3), and assistant nurse (4). Participants came from different units of care, including general psychiatric units (inpatient and outpatient care), psychosis units, child and youth psychiatry, psychiatric emergency units, and units for dependence care. Five of the 19 participants (2 nurses, 2 psychologists, 1 social worker) worked as head of unit, representing administrative staff according to SMS's classification. The participants' experience of working within mental health care varied between 1 - 41 years (mean $=20$ years). Fourteen participants had completed four web based modules and five participants had completed two out of four modules. Most participants had taken part in one (14) or two (4) group discussions. Only one had not done it at the time of the interview, but was included in the study since the other inclusion criteria were fulfilled.

\subsection{Data Analysis}

Data, consisting of the transcribed interviews and notes taken during the interviews, were analyzed using content analysis [22]. The transcripts were read several times to get an understanding of the whole. Meaning units relating to the research question were marked and coded. Codes were compared for similarities and differences and then abstracted into preliminary categories and subcategories. The transcripts were read again to see if the two latter were compatible with and covering the contents without leaving out important content relating to the study's aim. The results are presented with regards to SPiSS' different parts ("Web Based Modules”, "Group Discussions”) and their effect on participants ("Clinical Relevance and Use", "Communication and Climate").

\subsection{Ethical Considerations}

Care was taken to follow the Swedish Research Council's ethical principles "Good Research Practice” [23]. Participants were given written and oral information prior to the focus groups. All participants signed an informed consent. They were informed that participation was voluntary and that they could interrupt their participation without explanation or consequences for their work situation. Participants were informed about data being de-identified, handled confidentially and kept in a secure location.

\section{Results}

\subsection{The Web Based Modules}

\subsubsection{Repetition and Reminder vs New Knowledge}

The majority of participants describe the modules' contents as a structured repetition and useful reminder, while some parts represent new knowledge. The statistics are for instance thought provoking. The modules are seen as a good base rather than a full coverage of suicide prevention. A few participants experience the contents as too shallow and devoid of tangible tools for clinical praxis. Wishes for information on the suicide ladder, communication strategies and the handling of routines at the respective wards are expressed. Some participants miss a child and youth perspective, others see it although it can be further developed, especially considering today's use of social media.

Through repetition knowledge is revived, partly increasing awareness and wakefulness towards risk factors in patient meetings. Participants are reminded about the importance of enquiring into suicidal thoughts and plans. SPiSS is viewed as beneficial for new and less experienced staff, but even for more experienced staffs. Desensitization, routines or the feeling of already knowing the patient may lead to a risk of forgetting to enquire about suicidal ideation. The assessment instruments are appreciated, although participants pinpoint that all instruments are not used within the represented care units. Participants notice a somewhat increased awareness about the importance of documenting suicide assessments and the reasoning behind them more thoroughly to ensure patient safety.

\subsubsection{Convenient Format}

The web based format is appreciated for several reasons. The modules are delivered directly to participants at work and can be carried out at one's own pace, regardless of interruptions. The modules are described as well-structured and pedagogic. The knowledge tests require active and conscious reading and reflection, which is experienced as a positive challenge that can be repeated if needed. The modules are not experienced as too burdensome content or time wise. Nevertheless, even if the task is endorsed by the employer many participants feel that time is a problematic factor. The program takes time from other tasks. The fact that the group discussions are held in a different location, without disruptions, is thought of as advantageous.

\subsection{Discussion Groups}

\subsubsection{Different Types of Dialogue}

The extended time for dialogue and reflection is highly appreciated by most participants. The group discussions 
appear rewarding and informational, although their character differs amongst groups. Some meetings are described as an occasion to ventilate and discuss thoughts, feelings and reactions to suicide related questions, including personal narratives. The boundary between professional and personal reflections is sometimes blurred, which results in an opportunity for deep and eye-opening dialogues. Other groups keep the discussions closer to the filmed patient cases, which seem to work as base for worthwhile discussions. The meetings become an opportunity to discuss communication strategies, which are sought after due to their clinical relevance and use. Experienced flaws in the handling of patients in the filmed cases provoke reactions and discussions. The cases are seen as genuine and partly relevant and recognizable in relation to clinical practice. More cases combining both outpatient and inpatient care and with a child and youth perspective are wished for.

Most participants find the discussions interesting even if they don't result in practical solutions, but in more general dialogues about suicide related topics. Single participants describe practical solutions stemming from the discussions and entailing clear clinical use, such as ideas about how to use assessment instruments or other existing resources within their wards. Single participants view the discussions as totally devoid of practical use. They wish for dialogues about more concrete tools, such as communication strategies and a review of routines within the respective wards.

\subsubsection{Value of Mixed Groups and Leaders}

Most participants participated in groups with a mix of professions, even if they came from the same unit/ward. The mix of professions and units of care is seen as an advantage and a prerequisite for the exchange of knowledge and experiences, contributing to different perspectives on work with suicide assessments. A greater blend across units of care, especially between inpatient and outpatient care is seen as an accrued benefit.

The participants' experiences of the group leaders' contribution are varying. A certain level of leadership and steering seems meaningful and satisfying for the dialogues' contents and set-up. The role in more passive and invisible group discussion leaders is questioned. Own professional experience of psychiatric care does not seem to be decisive, even though it can be experienced as beneficial to lift clinically relevant questions throughout the meetings.

\subsection{Clinical Relevance and Use}

The fact that SPiSS should lead to a decrease of suicide attempts is questioned by most participants. Simultaneously many participants state that the update through the
SPiSS modules and group discussions has led to a partially enhanced knowledge, awareness and preparedness in relation to suicide prevention/assessments.

\subsubsection{Risk Factor Update}

The module about risk factors is the most often mentioned as being clinically useful. It works as an update of risk and protective factors and helps more clearly judge when a suicide evaluation is called for. Several participants believe that SPiSS has increased their awareness about risk factors in patient meetings, facilitating more structured assessments. SPiSS thus leads to a partially increased awareness, wakefulness, observation and comprehensive follow-up, even if these steps are part of routines. A few participants mention concrete examples where SPiSS had clinical value. The most frequent examples are connected to a higher awareness and visibility of risk factors in patient meetings. Needed measures were set in, such as requiring a physician's assessment and other care interventions such as a closer follow-up or a delayed leave from the ward. The participants describe that they felt strengthened in their assessments of patients.

\subsubsection{The Importance of Asking and Documenting}

Several participants describe SPiSS as an eye-opener. It reminds them about the importance of enquiring about patients' potential suicide thoughts or plans and about taking their worries about potential risks for self-destructive behavior in patients seriously. Enquiries about such thoughts may be forgotten in daily clinical work even if assessments belong to daily routines. SPiSS is a useful reminder. The importance of documenting the reasoning behind suicide assessments in more detail comes through as an important point for some participants. Several participants describe the assessment instruments as useful in the present context, even if they underline that they must be complemented with clinical dialogues with patients since instruments do not render a complete view of actual facts. One participant says that the use of instruments makes it easier and more natural to enquire about suicidal thoughts or plans when meeting patients.

\subsection{Effects on Communication and Climate}

When asking if SPiSS had an effect on communication, work climate or attitudes regarding suicide assessments at work mostly negative answers are given. It is pinpointed that suicide assessments are part of daily routines and hence continuously discussed; although participants from different units of care emphasize that assessments can be more (e.g. emergency units) or less (e.g. dependency care units) frequent depending on the type of unit. 
Generally no difference can be seen in the communication between colleagues or units of care, except for staff's occasional mentions of SPiSS, which represents a common point of reference. Single participants mention that SPiSS makes it easier to talk about suicide related issues at work, since it was slightly taboo and difficult to talk about before. Throughout the group discussions, it comes through that participants share comparable worries and thoughts about the difficulties related to suicide assessments across professions and units of care.

The fact that all staff within SMS is attending the program appears advantageous. It creates safety and a shared vision thanks to a common base that everyone is expected to have. The discussion groups contribute to a partly increased insight into and understanding of different units of care, even if most participants already work closely together with differing professions and have a good understanding of each others' work. A greater exchange between inpatient and outpatient care is wished for, for instance through suitable patient cases. It was thought that it may benefit the understanding, communication, care and patient outcome within and between the separate units of care.

Although most say that SPiSS does not affect their attitude towards suicide assessments, some participants point out that they are reminded about the fact that mental ill health is not outwardly visible; it can affect anyone regardless of background or social class. The statements are associated with the modules' photographs. Several participants mention that through discussions personal thoughts and experiences are touched upon, which is described as an eye-opener that makes mental ill health more visible in contexts beyond the professional one.

\section{Recommendation}

The majority of participants, with a few exceptions, can recommend the program with or without modifications. Suggestions made are a more visible child and youth perspective, more discussions of cases reaching over inand outpatient care, information about communication strategies and the suicide ladder, and concrete tools to overview routines on a ward level. Some participants consider that SPiSS is more suitable and rewarding for the social services, primary and outpatient care rather than for inpatient care and mental health services in general. Several participants forward that secretarial staff, not represented in the present sample, are questioning and critical towards their participation in SPiSS.

\section{Discussion}

\subsection{Discussion of Results}

The present evaluation study aimed at illuminating participants' experiences of SPiSS and its potential clinical relevance and use. This is thus not an effectiveness study, which would require a different design. The study shows that SPiSS contributes to a partly enhanced awareness and visibility of risk factors. It reminds of the importance of inquiring about suicide ideation/plans and documenting suicide assessments. Participants are strengthened in their assessments and worries about self-destructive behavior in patients. SPiSS seems to work as a valuable reminder and update, partially contributing to increased knowledge. This is in line with the Swedish government's priorities regarding enhanced competence within psychiatric care [24]. It also goes in line with SPiSS' keywords for suicide prevention, namely dare to ask, document, treat well, communicate and act. Several participants estimate that SPiSS is valuable for both less and more experienced staff, which may become desensitized and forget to enquire about suicidal thoughts or plans in patients already in care.

A product's usability is essential for its acceptance [25]. Most participants seemed positive towards the program and could see its potential clinical value. A minority was critical towards its value, which can be enhanced through further developments, e.g. a more developed youth perspective and more cases with a combined inand outpatient perspective. Critics found the program too shallow and lacking concrete tools on a ward level, which may affect implementation outcomes negatively. Nevertheless, the program is compulsory for all staff. Resistance on managers' level and resources [13] should thus not hinder SPiSS' implementation. Implementation strategies can be developed and measured on different levels relating to implementation, service, or client outcomes [26]. On the service level patient safety [26], a key goal for SPiSS, seems to be partially met through a slightly increased awareness, not the least through participants' appreciation of more thorough documentation to enhance patient safety.

The literature shows that individuals with habitual behaviors become less likely to act on new information and may even avoid information that challenges their current behavior [27]. Habits yield "tunnel vision" that can reduce the effectiveness of information-based interventions [28]. Nevertheless SPiSS comes across as a useful repetition that strengthens earlier beliefs, rather than challenges them. Recognizing and valuing participants' existing knowledge and experience has shown valuable for adult learning [29]. A supportive organizational infrastructure [30,31] and longer and more personalized periods of support from training experts [32] can also impact the success of health services activities. The recurrent discussion groups may contribute to a sense of continuousness and hence more efficacious implementation.

A minority found the discussion groups devoid of meaning and clinical value, while others appreciated 
them, whether they led to clinical solutions or not. Nevertheless, they contributed to an exchange of knowledge and experience, enriching participants' perspective on suicide assessments. The airing of personal narratives seemed like a positive experience. It may point to a need to ventilate thoughts and feelings connected to suicide, which briefing sessions at the wards may not entirely fulfill. Mentions of being reminded that mental ill health can affect anyone and of the occasional blurring of personal and professional contexts are also telling. One can hypothesize whether the barrier between private and professional roles is due to professionalism, to a shield to protect one's privacy and feelings, or if it is due to (self) stigma connected to mental ill health. A study on discrimination in people with mental illness has shown that patients frequently perceive discrimination from mental health care staff [33].

The web based format seems suitable, bringing the program to its users. It is well-structured, pedagogic and can be carried out at one's own pace despite interruptions. Convenience of access is a known advantage of the Internet [34]. The innovation's complexity should hence not be a barrier to adoption [13]. The knowledge tests require focused reading and reflection, which is experienced as a positive challenge. The web based modules and group discussions seem to fulfill different purposes. There can often be a tension between fidelity-the level to which a program is implemented as meant by its developers - and adaptation of interventions [35]. Fidelity to the program can be more or less ensured through the predetermined format of the modules and certification of units. However, the discussion groups appear to vary in terms of set-up and contents even if a prearranged format was planned. It may be due to the group leaders, but also to the participants themselves.

Raising awareness is one thing, acting another. To prevent suicide, we need to go beyond awareness towards acting and the integration of good habits. Knowledge, skills, attitudes and motivation may be satisfactory, but "old habits die hard" and contextual disturbances are more likely to lead to changing habits [28]. As seen in the present study, even experienced staff appreciated the program, which reminded them of the importance of enquiring into suicidal thoughts, making (good and bad) habits visible. Although education programs for primary care physicians have shown effective in recognizing and treating depression [3,9] it is not sufficient. Increased access to care is central [9] and the latter authors suggest additional studies including a combination of strategies on multiple levels of intervention (e.g. different target populations or levels of care, multiple key components) to evaluate potential synergistic effects of interventions to advance the knowledge base regarding suicide prevention. The experienced advantage of mixed discussion groups and cases including both in- and outpatient care in the present study speak for the advantage of targeting multiple populations/levels of care, with an appropriate adaptation of the intervention to fit its respective targets.

\subsection{Limitations}

Determining saturation in qualitative studies can be difficult. Nevertheless varied viewpoints were expressed. Data was rich and comparable across groups. At the end of each focus group the moderator summarized the main points discussed as a way of validating data, strengthening the results' reliability. The sample consisted of individuals with differing backgrounds, from diverse units of care and professions. It was representative of SMS in the sense that it entailed participants from diverse units of care within adult and youth psychiatry, although no representatives from forensic psychiatry participated in the study. All professions within SMS are not represented and their voices were hence not heard. Critical opinions from secretarial staff were thus forwarded "second hand", which may be interesting to explore in further studies.

The present study can only shed light onto participants' experiences within a relatively short time after their participation in SPiSS. Long-term epidemiological follow-up studies may shed light onto potential effects of the program on rates of suicide and suicide attempts. The study does not either tap into the client level, which could be measured through patient experiences and satisfaction with the program [26]. Interviews with patients may shed light onto essential "life-saving" factors in meetings with health professionals, even if it can be difficult to connect the results to SPiSS on a causal level.

\section{Conclusion}

The current study shows that SPiSS contributes to a partially increased awareness of risk factors, the importance of enquiring into suicidal thoughts and plans, and of documenting suicide assessments more thoroughly to enhance patient safety. Although the program's clinical relevance and use are questioned by some, the program works as a valuable repetition and reminder, both for less and more experienced staff. It can also be further developed to address unmet needs and enhance its clinical value.

\section{REFERENCES}

[1] World Health Organization, "The World Health Report 2003: Shaping the Future,” 2003.

[2] G. C. Patton, C. Coffey, S. M. Sawyer, R. M. Viner, D. M. Haller, K. Bose, et al., "Global Patterns of Mortality in Young People: A Systematic Analysis of Population Health Data," The Lancet, Vol. 374, No. 9693, 2009, pp. 881-892. 
http://dx.doi.org/10.1016/S0140-6736(09)60741-8

[3] J. J. Mann, A. Apter, J. Bertolote, A. Beautrais, D. Currier, A. Haas, et al., "Suicide Prevention Strategies: A Systematic Review,” JAMA, Vol. 294, No. 16, 2005, pp. 2064-2074. http://dx.doi.org/10.1001/jama.294.16.2064

[4] A. D. Lopez, C. D. Mathers, M. Ezzati, D. T. Jamison and C. J. Murray, "Global and Regional Burden of Disease and Risk Factors, 2001: Systematic Analysis of Population Health Data,” The Lancet, Vol. 367, No. 9524, 2006, pp. 1747-1757. http://dx.doi.org/10.1016/S0140-6736(06)68770-9

[5] J. B. Luoma, C. E. Martin and J. L. Pearson, "Contact with Mental Health and Primary Care Providers before Suicide: A Review of the Evidence," American Journal of Psychiatry, Vol. 159, No. 6, 2002, pp. 909-916. http://dx.doi.org/10.1176/appi.ajp.159.6.909

[6] U. A. Andersen, M. Andersen, J. Rosholm and L. Gram, "Contacts to the Health Care System Prior to Suicide: A Comprehensive Analysis Using Registers for General and Psychiatric Hospital Admissions, Contacts to General Practitioners and Practising Specialists and Drug Prescriptions," Acta Psychiatrica Scandinavica, Vol. 102, No. 2, 2000, pp. 126-134. http://dx.doi.org/10.1034/j.1600-0447.2000.102002126.x

[7] World Health Organization, "The World Health Report 2001-Mental Health: New Understanding, New Hope,” 2001.

[8] National Board of Health and Welfare, "Suicide Connected to Care," 2009.

http://www.socialstyrelsen.se/patientsakerhet/riskomrade $\mathrm{n} /$ sjalvmordianslutningtillvard

[9] C. M. van der Feltz-Cornelis, M. Sarchiapone, V. Postuvan, D. Volker, S. Roskar, A. T. Grum, et al., "Best Practice Elements of Multilevel Suicide Prevention Strategies: A Review of Systematic Reviews," Crisis: The Journal of Crisis Intervention and Suicide Prevention, Vol. 32, No. 6, 2011, p. 319.

[10] Department of Health and Social Affairs, "LSS-Law 1993:387,” 1993.

[11] National Board of Health and Welfare, "National Guidelines for Psychosocial Interventions in Patients with Schizophrenia-Support for Guidance and Management,” 2011, 2011-1-3.

[12] A. Pearson, H. Laschinger, K. Porritt, Z. Jordan, D. Tucker and L. Long, "Comprehensive Systematic Review of Evidence on Developing and Sustaining Nursing Leadership That Fosters a Healthy Work Environment in Healthcare," International Journal of Evidence-Based Healthcare, Vol. 5, No. 2, 2007, pp. 208-253. http://dx.doi.org/10.1111/j.1479-6988.2007.00065.x

[13] H. Brooks, D. Pilgrim and A. Rogers, "Innovation in Mental Health Services: What Are the Key Components of Success?” Implementation Science, Vol. 6, No. 1, 2011, p. 10. http://dx.doi.org/10.1186/1748-5908-6-120

[14] I.-L. Ramberg and D. Wasserman, "Suicide-Preventive Activities in Psychiatric Care: Evaluation of an Educational Programme in Suicide Prevention,” Nordic Journal of Psychiatry, Vol. 58, No. 5, 2004, pp. 389-394. http://dx.doi.org/10.1080/08039480410005954
[15] L. Wallin, "Knowledge Translation and Implementation Research in Nursing," International Journal of Nursing Studies, Vol. 46, No. 4, 2009, pp. 576-587. http://dx.doi.org/10.1016/j.ijnurstu.2008.05.006 PMid:18674761

[16] M. Donald, J. Dower and R. Bush, "Evaluation of a Suicide Prevention Training Program for Mental Health Services Staff,” Community Mental Health Journal, Vol. 49, No. 1, 2013, pp. 86-94.

http://dx.doi.org/10.1007/s10597-012-9489-y

[17] P. Skåne, “Kvalitetsbokslut 2012. Säker Hälsooch Sjukvård,” 2012.

[18] R. S. Barbour, "Making Sense of Focus Groups," Medical Education, Vol. 39, No. 7, 2005, pp. 742-750. http://dx.doi.org/10.1111/j.1365-2929.2005.02200.x

[19] D. L. Morgan, "Focus Groups,” Annual Review of Sociology, Vol. 22, No. 1, 1996, p. 129. http://dx.doi.org/10.1146/annurev.soc.22.1.129

[20] R. A. Krueger, "Analyzing \& Reporting Focus Group Results. Focus Group Kit 6,” Sage Publications Inc., London, 1998.

[21] R. S. Barbour and J. Kitzinger, "Developing Focus Group Research: Politics, Theory and Practice," Sage, London, 1999.

[22] U. H. Graneheim, B. Lundman, "Qualitative Content Analysis in Nursing Research: Concepts, Procedures and Measures to Achieve Trustworthiness," Nurse Education Today, Vol. 24 No. 2, 2004, pp. 105-112. http://dx.doi.org/10.1016/j.nedt.2003.10.001

[23] Swedish Research Council, “Good Research Practice,” 2002.

[24] Regeringskansliet, "PRIO Psykisk Ohälsa-Plan för Riktade Insatser Inom Området Psykisk Ohälsa 20122016,” 2012, p. 006.

[25] C. M. Allwood, "Människa-datorinteraktion. Ett Psykologiskt Perspektiv,” Studentlitteratur, Lund, 1998.

[26] E. K. Proctor, J. Landsverk, G. Aarons, D. Chambers, C. Glisson and B. Mittman, "Implementation Research in Mental Health Services: An Emerging Science with Conceptual, Methodological, and Training Challenges," Administration and Policy in Mental Health and Mental Health Services Research, Vol. 36, No. 1, 2009, pp. 2434. http://dx.doi.org/10.1007/s10488-008-0197-4

[27] B. Verplanken and W. Wood, "Interventions to Break and Create Consumer Habits,” Journal of Public Policy \& Marketing, Vol. 25, No. 1, 2006, pp. 90-103. http://dx.doi.org/10.1509/jppm.25.1.90

[28] P. Nilsen, K. Roback, A. Broström and P. Ellström, "Creatures of Habit: Accounting for the Role of Habit in Implementation Research on Clinical Behaviour Change,” Implementation Science, Vol. 7, No. 1, 2012, p. 53. http://dx.doi.org/10.1186/1748-5908-7-53

[29] M. S. Knowles, E. F. Holton and R. A. Swanson, “The Adult Learner: The Definitive Classic in Adult Education and Human Resource Development," 6th Edition, Elsevier/Buttrworth Heinemann, Amsterdam, 2005.

[30] P. Garside, “Organisational Context for Quality: Lessons from the Fields of Organisational Development and 
Change Management,” Quality in Health Care, Vol. 7, 1998.

[31] C. Jenkins, L. Baxter, J. Dowton, C. Gibbs and J. Partridge, "When Staff Training Isn't Enough: An Evaluation of a collaborative Approach,” International Journal of Language \& Communication Disorders, Vol. 33, No. S1, 1998, pp. 409-414.

[32] J. A. Kelly, A. M. Somlai, W. J. DiFranceisco, L. L. OttoSalaj, T. L. McAuliffe, K. L. Hackl, et al., "Bridging the Gap between the Science and Service of HIV Prevention: Transferring Effective Research-Based HIV Prevention Interventions to Community AIDS Service Providers," American Journal of Public Health, Vol. 90, No. 7, 2000, p. 1082. http://dx.doi.org/10.2105/AJPH.90.7.1082
[33] L. Hansson, S. Stjernswärd and B. Svensson, "Perceived and anticipated Discrimination in People with Mental Illness-An Interview Study,” Nordic Journal of Psychiatry, 2013, pp. 1-7.

[34] S. R. Cotten and S. S. Gupta, "Characteristics of Online and Offline Health Information Seekers and Factors That Discriminate between Them,” Social Science \& Medicine, Vol. 59, No. 9, 2004, pp. 1795-1806. http://dx.doi.org/10.1016/j.socscimed.2004.02.020

[35] L. Dusenbury, R. Brannigan, M. Falco and W. B. Hansen, "A Review of Research on Fidelity of Implementation: Implications for Drug Abuse Prevention in School Settings," Health Education Research, Vol. 18, No. 2, 2003, p. 237. http://dx.doi.org/10.1093/her/18.2.237 